\title{
Caracterización de las enfermedades raras, registradas en la Fundación Española de Beneficencia de Guatemala (1)
}

Dr. Leonardo Santa Luce*

Resumen

Se considera una enfermedad rara (ER) a todo aquel síndrome que tiene una prevalencia no mayor a cinco afectados por cada 10000 habitantes (2, párr. 3). El objetivo del presente estudio es caracterizar las enfermedades raras, desde un enfoque clínico y sociodemográfico. Se trata de un estudio descriptivo, retrospectivo, transversal y de caracterización de las ER registradas en la unidad de genética de un sanatorio privado, de enero 2013 a diciembre 2014. Los datos fueron recolectados desde los expedientes médicos, a través de un cuestionario clínico. La mediana de la edad de los pacientes identificados fue de 19 años, un $62 \%$ fueron de sexo femenino y el $8 \%$ reportó antecedente de ER. El $72 \%$ proviene del sector urbano y al 68 \% les tomó un tiempo mayor a cinco años, obtener su diagnóstico final después de, al menos tres consultas médicas. Las ER tienden a ser diagnosticadas clínicamente.

Solamente el $4 \%$ cuenta con acceso a un laboratorio genético. Los pacientes no tienen un tratamiento específico para curar la enfermedad, únicamente tratamiento sintomático. Dentro de las ER más frecuentes reportadas están: distrofia muscular de Dúchenme, síndrome de Turner y síndrome de Marfán. El 17 \% de los pacientes pertenece a una asociación de apoyo. Las ER representan un problema de salud pública, por su baja prevalencia y ser un campo desconocido para los servicios de salud en Guatemala.

Palabras clave: enfermedad rara, prevalencia, genética, salud pública.

\footnotetext{
* Médico y cirujano, magíster en Salud Pública con énfasis en Epidemiología y Gerencia por la Universidad Rafael Landívar. El presente texto del autor, revisado por Daniel Frade y Judith López de la Facultad de Ciencias de la Salud de la Universidad Rafael Landívar, resume el trabajo de tesis presentado para optar al grado de magíster en Salud Pública con énfasis en Epidemiología y Gerencia.
} 
This is a descriptive, retrospective, cross-sectional study of the characterization of $R D$ registered in the genetics department of a private hospital from January 2013 to December 2014. Data was collected through a clinical questionnaire from medical records. Median age is 19 years old among participants, generally from the urban sector (72\%). It takes more than five years to obtain their final diagnosis and $68 \%$ of the patients required at least three medical consultations. Only $4 \%$ have access to a genetic laboratory that confirms the diagnosis. Patients do not have a specific treatment to cure the disease, only symptomatic medications. The most frequent RDs reported are: Duchene Muscular Dystrophy, Turner Syndrome and Marfán Syndrome. Only 17 \% of patients are part of a support association. $R D s$ represent a public health challenge due to its low prevalence and an unknown field for health services in Guatemala.

Keywords: rare disease, prevalence, genetics, public health.

\section{Introducción}

Las enfermedades raras (ER), son un grupo de patologías que en la actualidad constituyen temas de discusión entre profesionales de salud a nivel mundial. Desde un enfoque conceptual se considera una enfermedad rara, todo aquel síndrome que tiene una prevalencia, no mayor a cinco afectados por 10000 habitantes (2, párr. 3 ).

La Organización Mundial de la Salud (OMS) estima más de siete mil el total de enfermedades o síndromes que reciben el término «raro», por su baja prevalencia en la población, aunque este número aumenta, conforme los avances de tecnología y técnicas permiten un mejor diagnóstico (1, p. 1).

Según la OMS el 7 \% de la población sufre una $E R$, lo que supone más de 42 millones en América Latina $(1$, p. 6). Tales enfermedades implican un grupo de problemas sobreañadidos, como la dificultad de realizar un diagnóstico rápido y certero; la falta de tratamientos adecuados e incluso la inexistencia de
Estas enfermedades, en su mayoría, se acompañan de disfunciones en varios órganos, patologías asociadas o discapacidades de cualquier otro tipo; por lo que los pacientes reciben atención segmentada de múltiples especialistas. Esto se refleja en un peregrinaje entre especialistas, mientras se diagnostica una ER. En su mayoría, son enfermedades de carácter degenerativo e irreversible que provocan incapacidades para tener una relación con su entorno físico o social (idem). Su baja prevalencia y dispersión determinan una baja repercusión social, pues en el grupo de pacientes que las padecen, la solidaridad no es patente. La relación costo beneficio no es interesante para el mercado, debido al uso restringido de los medicamentos y el número reducido de pacientes. Para concluir, no existe cobertura legal que fomente la investigación al respecto (ibidem, p. 2). Las ER reflejan un problema para la salud pública, debido a los efectos colaterales en lo social y psicológico; por desconocimiento, desconcierto y desorientación; por falta de protocolos y acceso a la información o por exclusión social, cultural y económica.

\section{Materiales y métodos}

Este estudio es de tipo descriptivo, retrospectivo y de corte transversal. La unidad primaria de análisis es el paciente con ER, registrado en un servicio de información y orientación (SIO) de la unidad de genética de un sanatorio privado de la Ciudad de Guatemala. Este servicio nace ante la necesidad de brindar capacitación, educación y orientación a los pacientes con estas patologías. La unidad de análisis comprende datos sociodemográficos y clínicos. El universo del estudio de 60 pacientes comprende niños, niñas, hombres y mujeres, de diversas edades $(N=60)$, registrados en la unidad de genética, entre los años 2013 y 2014. El criterio de inclusión fue: todos los pacientes registrados en la unidad de genética de un sanatorio privado de la Ciudad de Guatemala en esos años. Se consideró como criterio de exclusión, todos aquellos pacientes de la unidad de genética del sanatorio que no tienen una ER diagnosticada. 
Procedimiento y plan de análisis: se solicitó el acceso, a los datos de los pacientes registrados y atendidos, por la unidad de genética de un hospital privado, en el período del 1 de enero de 2013 al 31 de diciembre de 2014. Se revisaron expedientes clínicos de pacientes de la misma unidad y se aplicó, a los casos elegibles, un cuestionario conformado por preguntas sociodemográficas y clínicas. Los datos se trasladaron al programa estadístico SPSS para realizar tablas de frecuencia, gráficas y análisis de tipo estadístico descriptivo.

Aspectos éticos: categoría I (sin riesgo), utiliza técnicas observacionales, no se realiza ninguna intervención o modificación con las variables fisiológicas, psicológicas o sociales de las personas que participan en el estudio. La revisión de expedientes clínicos y otros documentos, no invaden la intimidad de la persona.

\section{Resultados}

Los pacientes incluidos en este estudio $(\mathrm{N}=60)$, representan el universo de registros en el período de tiempo del mismo, sin embargo, se excluyeron siete pacientes, por no tener una enfermedad rara, sino otra patología de diferente origen. Para cumplir con los criterios de exclusión del estudio, solamente se analizó un total de 53 pacientes.
Tabla 1. Indicadores sociodemográficas de pacientes con ER

\begin{tabular}{|c|c|c|c|}
\hline \multirow{2}{*}{\multicolumn{2}{|c|}{$\begin{array}{c}\text { Variables } \\
\text { sociodemográficas }\end{array}$}} & \multirow{2}{*}{$\begin{array}{l}\text { Indicadores } \\
\text { n }\end{array}$} & \multirow{2}{*}{$\begin{array}{c}(\mathrm{N}=53) \\
\text { porcentaje }\end{array}$} \\
\hline & & & \\
\hline \multirow{2}{*}{ Sexo } & hombres & 20 & 38 \\
\hline & mujeres & 33 & 62 \\
\hline \multirow{6}{*}{ Edad } & menores de 1 año & 2 & 4 \\
\hline & de 2 a 5 años & 3 & 6 \\
\hline & de 6 a 10 años & 2 & 4 \\
\hline & de 11 a 15 años & 5 & 9 \\
\hline & de 16 a 20 años & 20 & 38 \\
\hline & mayores de 21 años & 21 & 40 \\
\hline \multirow{2}{*}{ Procedencia } & rural & 15 & 28 \\
\hline & urbana & 38 & 72 \\
\hline \multirow{3}{*}{$\begin{array}{l}\text { Situación } \\
\text { laboral }\end{array}$} & empleado & 16 & 30 \\
\hline & desempleado & 7 & 13 \\
\hline & dependiente & 30 & 57 \\
\hline
\end{tabular}

Nota: número de pacientes por indicador sociodemográfico de los registrados en la unidad de genética de un hospital privado en Guatemala de enero de 2013 a diciembre de 2014. Fuente: Expedientes clínicos. 
Tabla 2. Indicadores clínicos de pacientes con ER

Variables
Clínico-terapéuticas

Indicadores $\quad(\mathbf{N}=\mathbf{5 3})$

n porcentaje

Antecedente médico

Si

4

$8 \%$

de ER

No

49

$92 \%$

Tiempo transcurrido
para el diagnóstico

Menos de

1 año

11

$21 \%$

De

Más de

2 a 5 años

7

$13 \%$

6 años

35

$66 \%$

$\begin{array}{llrr} & \text { De } 1 \text { a } 2 \text { consultas } & 17 & 32 \% \\ \text { Número de consultas } & \text { De } 3 \text { a } 4 \text { consultas } & 15 & 28 \% \\ \text { previas al diagnóstico } & \text { De } 4 \text { a } 6 \text { consultas } & 12 & 23 \% \\ \text { de ER } & \text { De } 7 \text { a } 8 \text { consultas } & 5 & 9 \% \\ & \text { Más de } 9 \text { consultas } & 4 & 8 \%\end{array}$

Laboratorios

Con laboratorios genéticos

2

$4 \%$

Sin laboratorios genéticos

51

$96 \%$

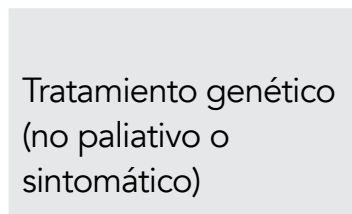

Pacientes con tratamiento

0

$0 \%$

(no paliativo o

farmacéutico

Pacientes sin tratamiento

53

$100 \%$

farmacéutico

Pacientes perteneciente a

9

$17 \%$

Asociaciones de grupos de apoyo apoyo

Pacientes no pertenecientes

44

$83 \%$ a grupos de apoyo

Nota: número de pacientes, por caso clínico, registrados en la unidad de genética de un hospital privado en Guatemala, de enero de 2013 a diciembre de 2014. Fuente: Expedientes clínicos. 


\section{Discusión y conclusiones}

Variables sociodemográficas

Sexo: las ER se presentan en ambos sexos por igual, sin embargo, existen muchas enfermedades genéticas asociadas, con mayor frecuencia al sexo femenino. La tabla 1 muestra que el $62 \%$ de los casos son de sexo femenino.

Edad: en el rango de las edades registradas se observa que la frecuencia más alta es la de mayores de 20 años, seguida por las edades 15 y 20 años. Las enfermedades raras aparecen, la mayoría de las veces, en edad pediátrica, por lo general, son anomalías congénitas. Sin embargo, la prevalencia es mayor en adultos que en niños, debido a la alta mortalidad de algunas enfermedades con malformaciones o genéticamente graves, y mal pronóstico de vida. El $40 \%$ de los casos registrados tienen más de 20 años. La mediana de la edad fue de 19 años, la media es de 15.4 años y la moda de 15 años.

Procedencia: el $72 \%$ de los pacientes $(n=38)$ procedían de las áreas urbanas, mientras que el $28 \%(n=15)$ provenía del área rural. La población estudiada pertenece a una unidad de genética de un sanatorio privado del área urbana con acceso exclusivo para personas que viven en la ciudad.

Condición laboral: los pacientes con ER, son con mayor frecuencia dependientes ( $57 \%, \mathrm{n}=30)$, es decir, no pueden subsistir por cuenta propia y reciben ayuda económica de otra persona. El 30 \% son personas económicamente activas $(n=16)$ y el resto desempleados

$(13 \%, n=7)$. Las ER, en la mayoría de sus casos, son enfermedades incapacitantes, por lo que la persona no puede trabajar en un empleo formal, en un área laboral específica. Esto genera un impacto en la economía de los pacientes, cuyo desarrollo personal y profesional se ve afectado y desfavorecido.

Variables clínicas

Antecedente genético: solamente el $8 \%$ de los casos $(n=4)$ dice / menciona tener algún antecedente médico asociado a una ER o a un trastorno genético hereditario. En el resto de pacientes (92\%, $n=49$ ), sus padres desconocen si estas ER eran parte de la herencia en el árbol genealógico de la familia y sus descendientes.

Tiempo en diagnosticar la ER: las ER se caracterizan por tardar de cinco a diez años en ser diagnosticadas. El $66 \%$ de los pacientes fue diagnosticado después de cinco años de presentar los primeros síntomas y el $21 \%$ obtuvo un diagnóstico menor a un año de sintomatología. El acceso a una unidad genética en un hospital privado favorece a los pacientes que nacen en dicha institución, por todas las técnicas diagnósticas y de tamizaje con las que se puede contar en el lugar.

Consultas previas al diagnóstico: se sabe que los pacientes con una enfermedad rara tienden a tener múltiples consultas, previo a su diagnóstico definitivo. A esto se le llama peregrinaje diagnóstico. El $68 \%$ de los pacientes $(n=41)$ acudieron a más de tres consultas para saber el diagnóstico de sus síntomas. El resto de pacientes obtuvo el diagnóstico entre la primera y segunda consulta $(32 \%, n=12)$.

Laboratorio genético: solamente el $4 \%$ de la población tiene acceso a un laboratorio genético que confirme su diagnóstico de ER. El resto solamente tiene acceso a un diagnóstico clínico de ER. Esto es debido al alto costo y a la inexistencia de métodos diagnósticos.

Tratamiento genético: ningún paciente tiene un tratamiento médico curativo para la ER diagnosticada. Todos los medicamentos utilizados para mejorar su calidad de vida, son de tipo paliativos o sintomático.

Asociaciones de apoyo: los pacientes con ER, por constituir poblaciones pequeñas, tienden a formar grupos de autoayuda; in embargo, únicamente el $17 \%$ de los pacientes $(n=9)$ pertenecen a uno. El resto se aísla, por miedo a la discriminación y marginación. Las ER más comunes son: distrofia muscular de Dúchenme, síndrome de Turner, dermatomiositis y síndrome de Marfán. Con menor frecuencia se presentan, el síndrome de Meniere, la distrofia muscular de Becker, y el síndrome de Sjorgen, entre otras. 


\section{Referencias}

1. Santa Luce L. Caracterización de las enfermedades raras registradas en la Fundación Española de Beneficencia de Guatemala[tesis en internet]. Guatemala:Universidad Rafael Landívar. 2017. Recuperado a partir de: http://recursosbiblio.url.edu.gt/tesisjrcd/2017/09/11/SantaLuce-Renato.pdf

2. Institut National de la Santé et de la Recherche Médical. Prevalencia de las enfermedades raras: Datos bibliográficos [internet]. France: Inf Per Orphanet. 2014 May; 2. Recuperado a partir de: http://www.orpha.net/orphacom/ cahiers/docs/ES/Prevalencia_de_las_enfermedades_raras_por_prevalencia_ decreciente_o_casos.pdf 